\title{
Treatment of mesial occlusion with forward displacement of the lower jaw in preschool children. Description of the clinical case
}

\author{
Eugenia S. Bimbas ${ }^{1}$, Anastasia S. Shishmareva ${ }^{1}$, and Eugenia V. Menshikova ${ }^{1}$ \\ ${ }^{1}$ Ural State Medical University, 620028, Repin str., 3, Yekaterinburg, Russia
}

\begin{abstract}
The scientific research is dedicated to studying of treatment problems of mesial malocclusion with the anterior mandible shift among preschool aged children (in the period of temporary dentition). The point of the study is to demonstrate the effect of treating mesial malocclusion associated with the mandible anterior shift and reverse overjet in a clinical case description. During the research of a clinical case long term results were analyzed. The method of treating mesial malocclusion associated with the mandible anterior shift and reverse overjet included usage of the original appliance. The appliance was also supposed to lead to harmonic development of a child's oro-facial area. The analysis showed advantages of the suggested original orthodontic appliance. The malocclusion was corrected in a short time ( 3 months), that let us develop physiological jaws and teeth arches relationships and to resolve mandible side shifting before the central incisors erupted. During the eruption permanent incisors take their place and establish the proper overjet, that creates physiological conditions for harmonic oro-facial development. The result is stable over time. The appliance is easy to use and to adapt by children and has a good fixation in oral cavity. Thus, the suggested orthodontic appliance could be recommended for treatment of mesial malocclusion associated with the mandible anterior shifting among preschool aged children.
\end{abstract}

\section{Introduction}

\subsection{The relevance of the study}

Nowadays the treatment of the mesial malocclusion among children is an important orthodontic problem. According to researches' data there is a growing number of patients with mentioned pathology in the latest decade. Moreover, the amount of patients with severe skeletal forms of mesial occlusion also increases. In the structure of dentition disorders the mesial malocclusion takes up to $16,9 \%$ due to late detection and the lack of 
aid system to this category of patients. Because of these reasons the malocclusion progresses with age. [1-5]. It is known, that there is no self-regulation of the bite among children with mesial malocclusion associated with the mandible anterior shift and reverse overjet during the skeletal growth. With no treatment it leads to the uncontrolled growth of lower jaw and the delay of the upper jaw growth, which result as a skeletal form of abnormality. The later patients come for an orthodontic treatment, the severer is the malocclusion. [6-10].

Today no orthodontic appliance, that could be used for treatment of the mesial malocclusion associated with the mandible anterior shift in preschool aged children, is mentioned in a special literature.

\subsection{Hypothesis}

Early orthodontic treatment helps to avoid a difficult long-lasting treatment of the mesial malocclusion among adults, and leads to a positive stable result.

\subsection{The point}

Demonstrating a clinical case with the preschool aged child having the mesial malocclusion associated with the mandible anterior shift and reverse overjet as an example of treatment method.

\subsection{Aims}

1. Analyzing immediate results of treatment of a patient with mesial malocclusion associated with the mandible anterior shift and reverse overjet.

2. Reviewing long term results of treatment of the patient with mesial malocclusion associated with the mandible anterior shift and reverse overjet.

\section{Materials and Methods}

The authors developed the orthodontic appliance - for treatment mesial occlusion with mandible shifting forward and reverse incisor overjet of pre-school children.

The appliance is made of certified materials which are used in orthodontic practice. These materials are without allergic reactions, without damages to oral tissue. The orthodontic appliance was used for a child, parents signed consent to medical intervention and an agreement to use this apparatus for the child.

\subsection{Consider the clinical case}

The child B's parents consulted the orthodontist's doctor and complained about the violation of facial aesthetics (picture 1) and violation of the chewing function. The child is 4 years old. Anamnesis of life (or anamnesis vitae): there aren't any factors that have influenced the development. Anamnesis morbi: according to parents words they have noted their child has incorrect closing of teeth after slitting of all milk teeth. They haven't applied for orthodontic help before. The clinical examination. External examination: the face is symmetrical, nasal folds are smoothed, the middle and lower parts of the face are proportional, the profile is straight. Upper lip is flattened. The opening of mouth is freely, full, the type of breath - nose. In the oral cavity there are milk occlusion; the distal 
surfaces of the second milk molars of the mesial stage to the right and left; reverse incisor overjet, trims of the upper and lower tooth rows (Fig.1 (a)).

The comprehensive survey was conducted with additional diagnostic techniques of the patient B: panoramic x-ray, analysis of control diagnostic models. Evaluation of clinical functional test (test of Illinoy-Markosyan) showed a mandible shifting forward: the patient can set incisors in direct connection [4, 11].

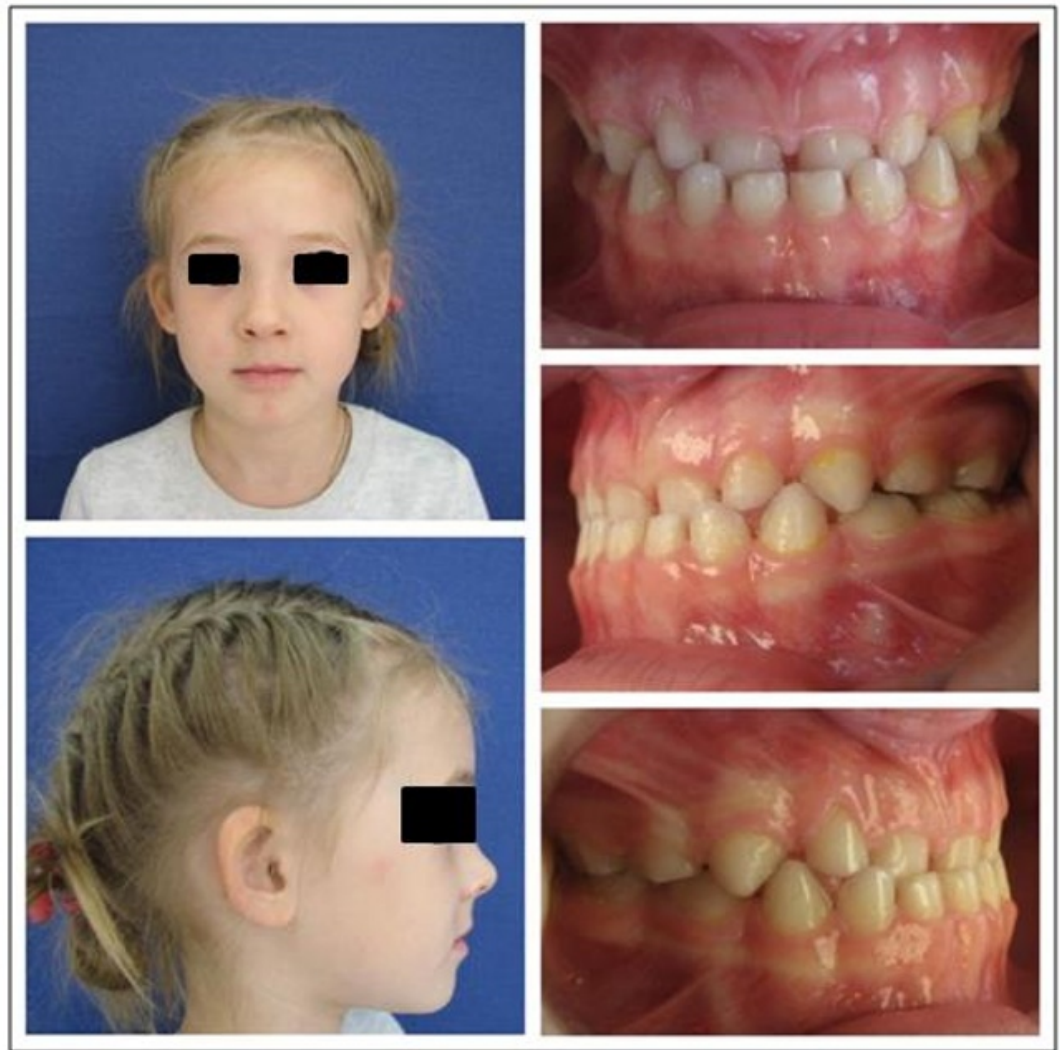

Fig. 1(a). Patient B. is before treatment

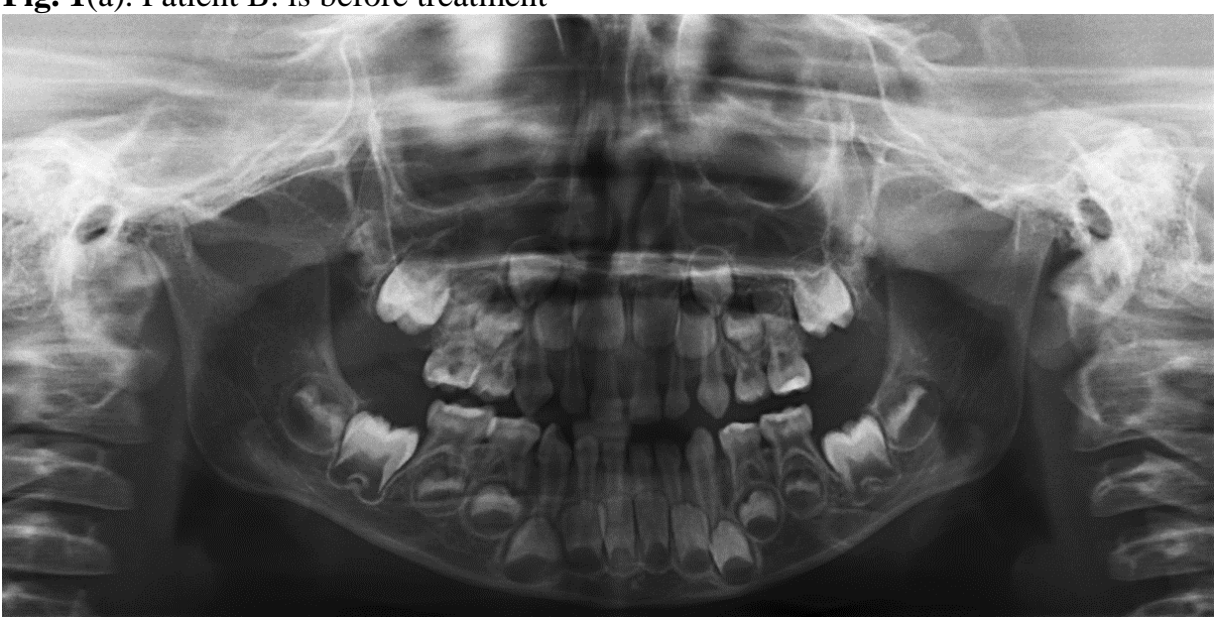

Fig. 1 (b). Panoramic x-ray before treatment. 
At the presented panoramic x-ray (Fig.1 (b)) of jaws before treatment: bone cloth with a clear drawing; bone beams are visible evenly and clearly at all sites; cortical bone is visible clearly and continuous at all sites of the body of jaw and the jaw branches; the jaw branches are symmetric; maxillary sinuses are pneumatic; homogeneous. There are all permanent teeth germs besides 1.8, 2.8, 3.8, 4.8 teeth germ; uniform physiological resorption of milk roots teeth. There isn't disorder of jaw growth: trims absent without permanent teeth 3 germs, individual indicator of permanent teeth germ formation is equivalent to the age norms.

There was a diagnosis: "mesial malocclusion, reverse incisor overjet, forced forward mandible displacement".

K07.2 Anomalies of dental arch relationship. Mesio-occlusion.

\subsection{Treatment plan}

A complex treatment plan is created and confirmed based on the medical examination:

Pre-orthodontic preparation: professional oral hygiene, oral cavity's sanitation.

Orthodontic treatment:

Phase I. Early orthodontic treatment, aimed to resolving the forced mandible anterior shift and creating optimal conditions for a physiological jaws' growth. The original orthodontic appliance was used for treating. The suggested appliance (Fig. 2 (a) - the right side view, Fig. 2 (b) - the frontal view) consists of an upper jaw cape (1) and a lower jaw cape (2). Besides, there are some hooks embodied into the plastic mass in the area of upper second milk molars and lower milk canines (3). Both caps are bonded to each other by elastic rings (4), which are attached to the hooks. These elastics can develop forces up to 50-100 $\mathrm{g}$ on the each side.

Phase II: observing the results.

\subsection{Dynamics of the orthodontic treatment}

1. Appointment: taking alginate impressions of both teeth arches and determination of the constructive overbite (position of marginal incisors contact).

2. Appointment: adjustment of the orthodontic appliance with the original construction. During the adjustment of the appliance in the oral cavity these parameters were checked: the accurate fit of the upper and lower caps to the teeth; the lack of contact between the appliance and soft tissues; the availability of sliding between the caps. Then the appliance was set on the upper and lower teeth using a glass-ionomer cement. The patient and her parents were taught oral hygiene with the appliance. It was recommended to use maxilla-

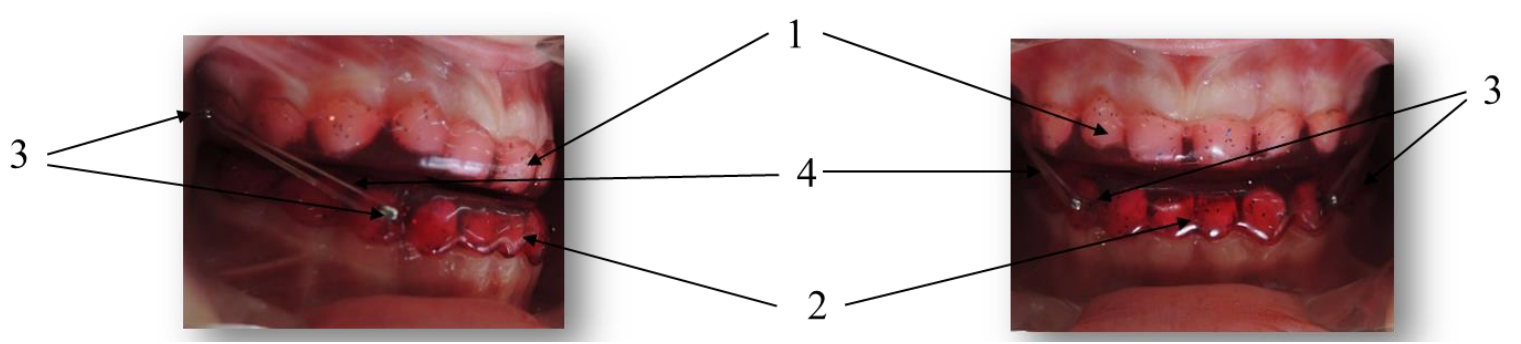

mandibular elastics (elastics "Zoo" by ORMCO: "Chipmunk"). Wearing of the elastics 24 hours a day with rings' change every 12 hours. 
Fig. 2 (a).

Fig. 2 (b).

3. Appointment: the patient is adjusted to the appliance, wears elastics regularly. It is recommended to wear the appliance for 2 months within the further use of the elastics.

4. Appointment: appliance and glass-ionomer cements are removed, polishing, teeth fluoridation (Fig. 3). At the follow-up appointment 3 months later it is determined that the lower jaw position and teeth arches relationship are physiologically right. Overbite $0.5 \mathrm{~mm}$, overjet $0.2 \mathrm{~mm}$.

The I phase of treatment lasted for 3 months.

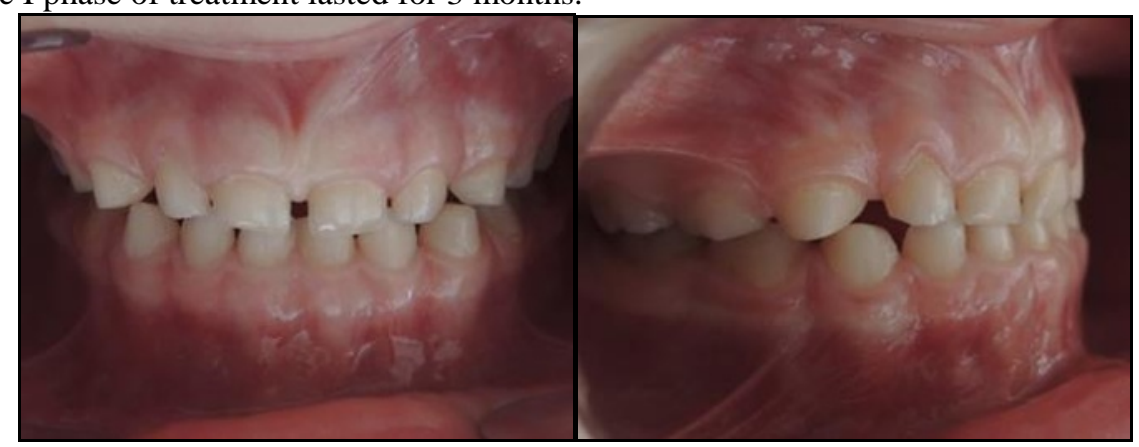

Fig. 3. Patient after the treatment.

\section{Results and Discussion}

Therefore, the orthodontic appliance for the treatment of mesial malocclusion with anterior mandible shift and reverse overjet eliminated the malocclusion as quickly as possible (3 months). There was correct overjet; lower jaw removed from forced forward position.

The right dentition at 7 years old shows about positive impact of provided treatment. The early removal of overjet allows to the permanent incisures to be mounted in the correct overlap. Observations over a period of 1-5 years show harmonious development of the maxillofacial region.

Monitoring has been conducting for 5 years since the moment of lifting the appliance. In the examination of a patient aged 8: the face is slightly convex profile; the first molars stage to Angle class I; correct overbite of permanent incisors $(1 \mathrm{~mm})$; presence of overjet (1 $\mathrm{mm}$ ) - this indicates a physiological change of teeth (Fig.4). 

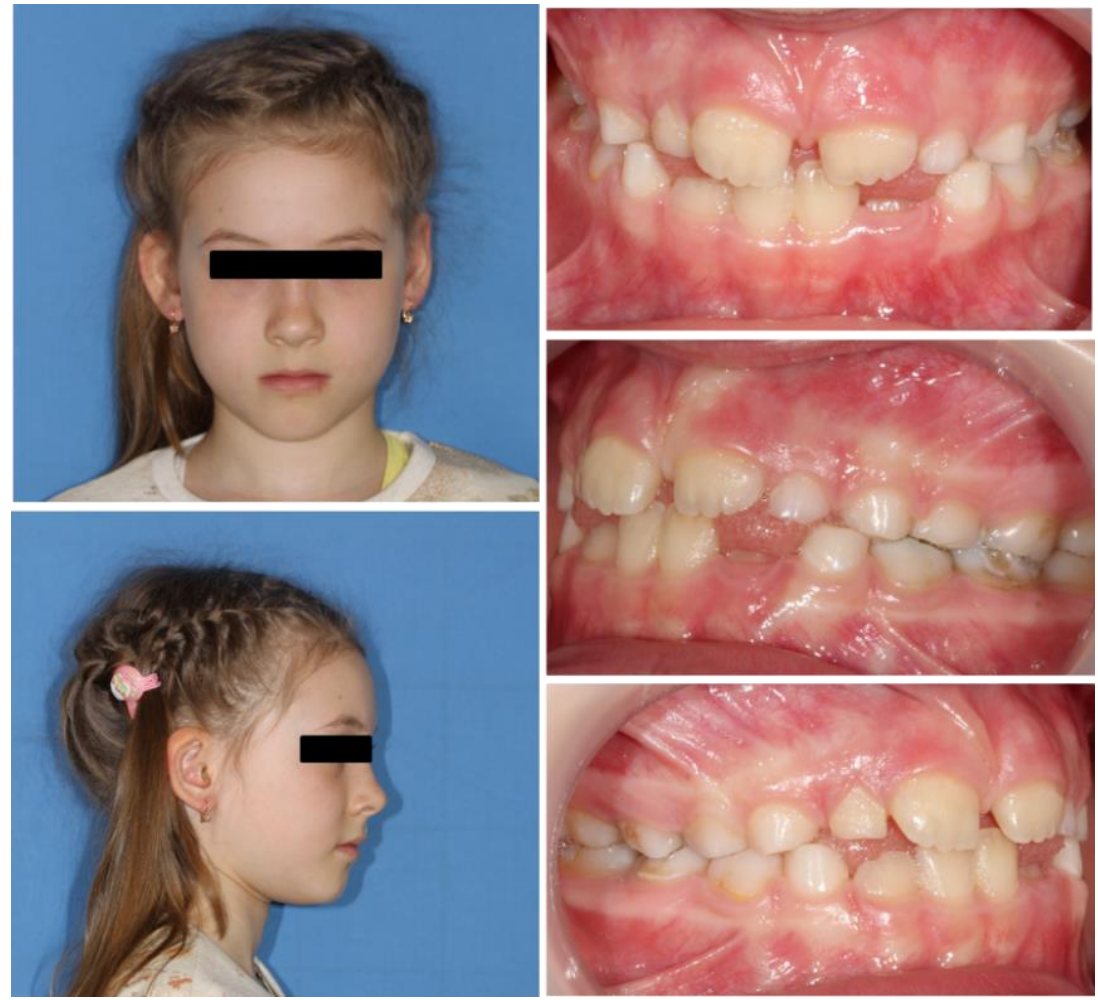

Fig. 4. Patient B. are in the dynamic observation phase (4 years after treatment).

\begin{tabular}{|c|c|c|}
\hline Indicator & Standard & After treatment \\
\hline SNA & $\mathbf{8 2 + / - 2}$ & $\mathbf{8 0}$ \\
\hline SNB & $\mathbf{8 0 + / - 2}$ & 78 \\
\hline ANB & $\mathbf{2 + / - 2}$ & $\mathbf{2}$ \\
\hline NSL/ML & $\mathbf{3 2 + / - 5}$ & $\mathbf{3 0}$ \\
\hline NSL/NL & $7+/-2$ & 10 \\
\hline NL/ML & $\mathbf{2 5 + / - 3}$ & $\mathbf{2 0}$ \\
\hline ILS/NL & $115+/-5$ & 118 \\
\hline ILi/ML & $90+/-5$ & 94 \\
\hline cm-sn-ls & $112+/-2$ & 115 \\
\hline gl-sn-pg & $\mathbf{1 2 + / - 2}$ & 9 \\
\hline
\end{tabular}

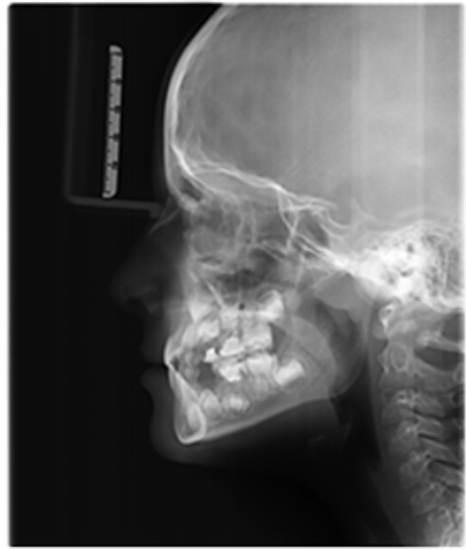

Fig. 5. Lateral cephalometric $x$-ray of patient $B$ after the treatment.

Analysis of the lateral cephalometric x-ray of patient aged 9 years shows: the first skeletal class; normal position base of jaws; normal incisures position; convex profile. This is indicative of the harmonious development of the maxillofacial area. 


\section{Conclusion}

The research showed the effectiveness of the author's apparatus for early treatment of mesial occlusion with mandible shifting forward and reverse incisor overjet of pre-school children. The apparatus made it possible to achieve the correct relationship of teeth rows and harmonious development of the maxillofacial area to distant timing of observation.

\section{References}

1. N.V. Myagkova, Gnatic forms of mesial occlusion: improving methods of diagnostics and treatment of patients of various ages, Autoabstract of the dissertation for the degree of doctor of medicine (Yekaterinburg, 2017)

2. N.V. Myagkova, Ural medical journal, 07(140), 38-43 (2016)

3. A. Kumar, A. Rohilla, P. Tandon, A. Nager, Int J Orthod Milwaukee, 25(2), 41-5 (2014)

4. P. Flis, V. Filonenko, N. Doroshenko, Georgian Med News, Oct; (271), 18-23 (2017)

5. Rohit A. Minase, Wasundhara A. Bhad, Umal H. Doshi, Prog Orthod., 20(1), 14 (2019) doi: 10.1186/s40510-019-0266-0

6. M.A. Postnikov, D. A. Trunin, N.V. Pankratova, A.M. Nesterov, M.I. Sadykov, S.A. Ostankov, Stomatology of children's age, 97(1), 59-62 (2018)

7. M. Foersch, C. Jacobs, S. Wriedt, M. Hechtner, H. Wehrbein, Clin Oral Investig., 19(6), 1181-92, (2015) doi: 10.1007/s00784-015-1478-4

8. S.A. Al-Mozany, O. Dalci, M. Almuzian, C. Gonzalez, N.E. Tarraf, M. Ali Darendeliler, Prog Orthod., 18(1), 40 (2017) doi: 10.1186/s40510-017-0192-y

9. Robinsón-Andrés Castrillón-Marín, Diana-María Barbosa-Liz, Carlos-Martin Ardila, J Clin Exp Dent., 11(7), e665-e669, (2019) doi: 10.4317/jced.55939

10. Letizia Perillo, et al, European Journal of Orthodontics, 38(1) 51-56 (2016) doi:10.1093/ejo/cjv010

11. N.V. Myagkova, Journal Problems of dentistry, 4, 67-71 (2013) 